\title{
Solved? The reductive radiation chemistry of alanine
}

\author{
Ewald Pauwels $^{1 * \#}$, Hendrik De Cooman ${ }^{1}$, Michel Waroquier ${ }^{1}$, Eli O. Hole ${ }^{2}$, Einar \\ Sagstuen $^{2}$ \\ ${ }^{I}$ Center for Molecular Modeling, Ghent University, Technologiepark 903, B-9052 \\ Zwijnaarde, Belgium \\ QCMM - alliance Ghent-Brussels, Belgium \\ ${ }^{2}$ Department of Physics, University of Oslo, P.O. Box 1048 Blindern, N-0316 Oslo, Norway \\ *Towhom all correspondence should be addressed.E-mail: ewald.pauwels@UGent.be \\ * Present address: Information and Communication Technology Department, Ghent University, \\ Krijgslaan 281 S9, B-9000 Ghent, Belgium
}

\begin{abstract}
The structural changes throughout the entire reductive radiation-induced pathway of L- $\alpha$-alanine are solved on an atomistic level with the aid of periodic DFT and nudged elastic band (NEB) simulations. This yields unprecedented information on the conformational changes taking place, including the protonation state of the carboxyl group in the "unstable" and "stable" alanine radicals and the internal transformation converting these two radical variants at temperatures above $220 \mathrm{~K}$. The structures of all stable radicals were verified by calculating EPR properties and comparing those with experimental data. The variation of the energy throughout the full radiochemical process provides crucial insight in the reason why these structural changes and rearrangements occur.

Starting from electron capture, the excess electron quickly localizes on the carbon of a carboxyl group, which pyramidalizes and receives a proton from the amino group of a neighboring alanine molecule, forming a first stable radical species (up to $150 \mathrm{~K}$ ). In the temperature interval $150-220 \mathrm{~K}$, this radical deaminates and deprotonates at the carboxyl group, the detached amino group undergoes inversion and its methyl group sustains an internal rotation. This yields the so-called "unstable alanine radical". Above $220 \mathrm{~K}$, triggered by the attachment of an additional proton on the detached amino group, the radical then undergoes an internal rotation in the reverse direction, giving rise to the "stable alanine radical", which is the final stage in the reductive radiation-induced decay of alanine.
\end{abstract}




\section{Introduction}

L- $\alpha$-alanine, ${ }^{+} \mathrm{NH}_{3}-\mathrm{CH}\left(\mathrm{CH}_{3}\right)-\mathrm{CO}_{2}{ }^{-}$, is one of the most exhaustively studied molecules in the field of radiation chemistry. Being an essential amino acid, it is a simple model system to investigate the action of radiation on proteins. One of the most harmful effects of ionizing radiation is the formation of primary oxidative (one-electron loss) and reductive (one-electron gain) free radicals, which subsequently decay and transform into more stable (radical) fragments ${ }^{1}$. In alanine, these processes have been examined to great length in different phases and at various temperatures, both in experiments $^{2-22}$ and theoretical investigations. ${ }^{23-29}$ Yet, in spite of its structural simplicity, alanine's radiation chemistry has proven elusive.

Because of the relative stability of radicals in the solid state, a host of studies have focused on this phase, employing mostly Electron Paramagnetic Resonance (EPR) spectroscopy. Dating back to the fifties ${ }^{30}$, a stable radical was detected using EPR in X-irradiated single crystals of alanine - dubbed "Stable Alanine Radical" $(S A R)$. This radical is the dominant final stage in the decay of the radiation-induced radical species in alanine, but the prevailing view on its structure was recently proven to be incorrect. In our 2010 study $^{23}$ we show that the $S A R$ is composed of a negatively charged, deaminated $\mathrm{CH}_{3} \mathrm{C} \cdot \mathrm{HCO}_{2}{ }^{-}$radical and an associated $\mathrm{NH}_{4}{ }^{+}$ ammonium ion close by. This radical is not protonated at the carboxyl side, as is frequently considered. ${ }^{11-18}$ Information on the primary radiation-induced radicals, leading up to the $S A R$, has been obtained in EPR experiments conducted at lower temperatures, ${ }^{2-9}$ frequently also identifying other, minor radical species. ${ }^{17,19-22}$ In their 2004 review $^{31}$, Sagstuen et al. assemble the wealth of available data and outline the oxidative and reductive pathways through which the observed radical species transform into one another, solving a number of issues but also leaving some unresolved.

One important unresolved issue concerns the precise formation of the $S A R$ through the reductive pathway. As illustrated in Scheme 1, this pathway is commonly considered to entail electron capture by a pristine $\mathrm{L}-\alpha$-alanine molecule, resulting in an anionic radical species (a0a) in which the unpaired electron quickly localizes at the carboxylic carbon (a1a), followed by protonation (a2a). The first two transient species (a0a and a1a) have not been observed directly in EPR experiments, but measurements have been made for the latter species (a2a) up to $150 \mathrm{~K}^{2-7}$ At higher temperatures, this radical species deaminates, generating a3a, which has been characterized with EPR and found to be stable up to $200-220 \mathrm{~K}^{8,9}$ This species has often been called the "unstable alanine radical". Above $220 \mathrm{~K}$, it gradually transforms ${ }^{32}$ into the $S A R$ (species a4a) through an intramolecular rearrangement. Yet, the exact nature of this rearrangement from a structural point of view is entirely unclear, as is the reason why this rearrangement occurs.

In this work, we apply theoretical calculations - using Density Functional Theory (DFT) - to shed light on the molecular transformations and rearrangements taking place throughout the entire reductive radiation-induced pathway of alanine, starting from a0a and ending at a4a. By comparing calculated EPR properties with experimental data, all intermediate radical species throughout the pathway are positively identified. Specific attention is paid to the treatment of the molecular environment in all simulations, as this proved to be of the utmost importance in our previous publication. ${ }^{23}$ In addition, we determine the variation of the potential energy, yielding unprecedented insight into the reason why this radiation-induced pathway occurs. 


\section{Computational Details}

Calculations were performed in a periodic approach with the $\mathrm{CP} 2 \mathrm{~K}$ software ${ }^{33}$ on a crystallographic unit cell of alanine ${ }^{34}$ that was duplicated along the $<a>$ and $<\mathrm{c}>$ directions, yielding a supercell [2ab2c] of dimensions $12.050 \times 12.324 \times 11.566 \AA^{3}$. In this way, any interaction of a radical with its periodic images was essentially prevented. This supercell was subject to energy minimization ${ }^{35}$ and then a negative charge was added, arriving at the structure of radical model a0a. The subsequent radical models in the radiation-induced pathway were found by altering this starting structure in accordance with Scheme 1, followed by energy minimization. For the $\mathbf{a 4} \mathbf{a}^{1}$ and $\mathbf{a 4} \mathbf{a}^{2}$ models (see Figure 2), the supercell was rendered neutral by protonation. Whenever possible, the structure of a radical model was verified by calculating EPR properties and comparing those with experimental data.

To examine the conformational changes between consecutive minima and obtain an estimate of the associated energy profile, reaction paths were simulated with the nudged elastic band (NEB) method. ${ }^{36,37}$ In this approach, the reaction pathway between two points is represented by a series of intermediate configurations (replicas) that are connected by harmonic spring forces. Optimization of this system yields a close estimate of the minimum energy path connecting the two points. We used 16 replicas and a combined steepest descent - DIIS optimizer. ${ }^{38}$ The initial guess for the band was generated by linear interpolation between optimized radical structures. The IT-NEB algorithm ${ }^{37}$ was then applied for several cycles, followed by CI-NEB ${ }^{36}$ until sufficient convergence was reached. The replica with maximal energy along the NEB path was taken as an approximation to the transition state. In a recent study, ${ }^{39}$ a similar protocol was applied to examine radiation-induced conformational changes.

The BLYP density functional ${ }^{40,41}$ was used in all simulations. In energy minimizations and NEB runs, the Gaussian and plane waves (GPW) dual basis set method $^{42}$ was used, employing a TZVP triple- $\zeta$ Gaussian basis set ${ }^{43}$ and plane waves (400 Ry density cut-off) with GTH pseudopotentials. ${ }^{44,45}$ For the calculation of EPR properties (g- and hyperfine coupling tensors), we relied on recent implementations in the $\mathrm{CP} 2 \mathrm{~K}$ code, ${ }^{46,47}$ employing the all-electron Gaussian and augmented plane wave (GAPW) method. ${ }^{48}$ The density cut-off for the auxiliary plane wave basis set was 200 Ry and the all-electron TZVP basis ${ }^{49}$ was used. This dual methodology has proven successful in various other studies. ${ }^{23,39,50,51}$

\section{Results and Discussion}

Combining the results of all minimizations and NEB runs, a comprehensive picture emerges of the processes taking place during the radiation-induced formation of the $S A R$. An overview of the variation of the potential energy is given in Figure 1 and the three-dimensional structures of all reported minima are shown in Figure 2. In the supplementary information, an animation illustrates the complete process (created using $\mathrm{VMD}^{52}$ ).

\section{Conversion of a0a to a $2 a$}

Structure a0a corresponds to the crystal structure, but the supercell is anionic and paramagnetic, representing a small portion of the lattice where electron capture has just occurred. At this stage the spin density is distributed across the supercell, mainly on the oxygens. Upon energy minimization, the spin density quickly localizes on 
atom $\mathrm{C} 1$ of one of the alanine molecules. At the same time, the corresponding carboxyl group pyramidalizes - either 'up' or 'down' - to accommodate the unpaired electron, as has been found in other studies. ${ }^{53-55}$ The $\mathbf{a 1 a ^ { \text { up } }}$ and $\mathbf{a 1 a}^{\text {down }}$ models indicated in Figure 1, however, do not constitute minima yet.

Consistent with Scheme 1, the carboxyl group becomes protonated before a local minimum on the potential energy surface is found. As is clear from Figure 2, three protonation routes are possible from nearby ammonium groups: one towards oxygen $\mathrm{O} 1(a)$ and two towards $\mathrm{O} 2(b$ and $c$ ). All possibilities give rise to stable radicals, but with considerable variation in their final potential energy. At the 'up'side, protonation on $\mathrm{O} 2$ via route $b\left(\mathbf{a}^{\mathbf{u}} \mathbf{a}^{\mathbf{u p}}\right)$ yields the lowest potential energy. At the 'down'-side, the lowest energy is achieved via route $a$, protonating oxygen $\mathrm{O} 1$ $\left(\mathbf{a}^{\text {down }}\right)$; route $b$ results in a structure that is only slightly higher in energy $(\sim 3$ $\mathrm{kJ} / \mathrm{mol}$ ). These two 'down' radicals have considerably lower energies than all three radicals on the 'up'-side, making their involvement a thousand-fold more probable (Boltzmann statistics at $150 \mathrm{~K}$, the temperature up to which species a2a can be observed experimentally). Similarly, protonation on the 'down'-side via route $a$ is tenfold more likely than via route $b$. These energetic considerations concur with the observations made by Miyagawa et al. ${ }^{2}$ that protonation only occurs at one of the carboxyl oxygens.

\section{Spectroscopic identification of a2a}

Convincing assignment of this structure comes from a comparison of measured EPR data for species a2a and calculated magnetic resonance properties for model $\mathbf{a} 2 \mathbf{a}^{\text {down }}$ in Table 1. Despite a few discrepancies, our calculations agree reasonably well with the measurements. Perhaps most compellingly, this structure is the only one that matches the overall pattern of isotropic hyperfine couplings and g-tensor values of the experiment. This becomes apparent from a similar comparison between theory and measurements for all other stable radicals on either 'up' or 'down' side, given in Table SI-1 of the supplementary information.

Considering that it has the lowest potential energy and that there is an agreement for the magnetic resonance properties, it is highly likely that radical model $\mathbf{a} 2 \mathbf{a}^{\text {down }}$ corresponds to radical species $\mathbf{a 2 a}$ in the reductive radiation-induced pathway of alanine. Intriguingly, the ammonium proton that transfers to the carboxyl oxygen in this structure is the most distant in the undamaged crystal structure: $1.82 \AA$ (route $a$ ) versus $1.70 \AA$ (route $b$ ) and $1.80 \AA$ (route $c$ ). This illustrates that radiation chemistry, more than often, remains elusive for physical or chemical intuition.

\section{Conversion of a 2 a to a3a}

At temperatures above $150 \mathrm{~K}$ the a2a radical species deaminates, generating radical a3a. To investigate the formation of this latter species, gradual $\mathrm{C} 2-\mathrm{N}$ bond elongation in structure $\mathbf{a 2} \mathbf{a}^{\text {down }}$ was imposed, followed by regular energy minimization, from which we established three stable radical models: $\mathbf{a 3 a} \mathbf{a}^{\mathbf{1}}, \mathbf{\mathbf { a 3 a } ^ { 2 }}$ and $\mathbf{a} \mathbf{3} \mathbf{a}^{3}$. With the aid of NEB simulations, the minimal energy paths connecting these stable radicals were further explored. Below, we describe the sequence of events in those paths. The results are also represented in Figures 1 and 2.

Radical model $\mathbf{a} 2 \mathbf{a}^{\text {down }}$ first undergoes detachment of its ammonium group and deprotonation of the carboxyl oxygen, leading to radical model $\mathbf{a} 3 \mathbf{a}^{\mathbf{1}}$, which is about $10 \mathrm{~kJ} / \mathrm{mol}$ more stable. These processes seem to happen simultaneously since NEB refinement did not find any intermediate minima. Deamination is clearly controlling the reaction: a huge barrier of about $50 \mathrm{~kJ} / \mathrm{mol}$ needs to be surpassed. This explains 
the relative stability of species a2a up to temperatures of $150 \mathrm{~K}$, since sufficient thermal energy is required to make the transition. Subsequently, the detached $\mathrm{NH}_{3}$ group undergoes inversion. Surpassing the associated small barrier yields a tremendous exothermic benefit: radical model $\mathbf{a 3 a}^{\mathbf{2}}$ is almost $20 \mathrm{~kJ} / \mathrm{mol}$ more stable than $\mathbf{a} 3 \mathbf{a}^{\mathbf{1}}$. Finally, the methyl group of the radical is rotated away from the nearby ammonia, leaving $\mathrm{H} \alpha$ in a position just below $\mathrm{NH}_{3}$. This internal rotation about the $\mathrm{C} 1-\mathrm{C} 2$ axis requires considerable energy, but $\mathbf{a 3 a}^{\mathbf{3}}$ is again more stable than its predecessor by close to $20 \mathrm{~kJ} / \mathrm{mol}$. We also attempted to reverse the order of ammonia inversion and internal rotation to generate this last radical model, but much higher energy barriers were associated with this order of events.

The potential energy plot in Figure 1 clarifies why none of the intermediate radicals ( $\mathbf{3} 3 \mathbf{a}^{\mathbf{1}}$ or $\mathbf{a} 3 \mathbf{a}^{\mathbf{2}}$ ) have been detected in experiment: once the barrier between $\mathbf{a} 2 \mathbf{a}^{\text {down }}$ and $\mathbf{a 3 \mathbf { a } ^ { 1 }}$ is surpassed, enough potential energy is released to overcome the other, much smaller barriers and the system remains trapped at the $\mathbf{3} 3 \mathbf{a}^{\mathbf{3}}$ end point.

\section{Spectroscopic identification of a3a}

A comparison between the calculated EPR properties of radical model $\mathbf{a 3 a}^{\mathbf{3}}$ and measurements for species a3a at $220 \mathrm{~K}$ is shown in Table 2. The main spectroscopic features are one $\alpha$-type and one $\beta$-type hyperfine coupling corresponding, respectively, to the $\mathrm{H} \alpha$ proton and the protons of the methyl group, which at $220 \mathrm{~K}$ rotates freely about the $\mathrm{C} 2-\mathrm{CH}_{3}$ axis. Our calculations yield similar spectroscopic features for model $\mathbf{a 3 a}^{\mathbf{3}}$, with anisotropic hyperfine couplings matching very well with their experimental counterparts and principal directions that are mostly reproduced within $7^{\circ}$. In previous works, ${ }^{56}$ agreement of the principal directions has proven critical to establish the accuracy of a proposed radical model. The isotropic hyperfine coupling for the methyl proton is very good, but that for $\mathrm{H} \alpha$ is off by 17 MHz. This is not uncommon since $\alpha$-type couplings are known to be quite susceptible to level-of-theory and temperature effects. ${ }^{57}$

Several long-range dipolar couplings (included in Table 2) can also be assigned in our model structure. $\mathrm{H}(\mathrm{C}) 1$ and $\mathrm{H}(\mathrm{C}) 2$ are two methyl protons of an alanine molecule positioned right underneath $\mathrm{C} 2$ of the radical (see Figure SI-1 in the supplementary information). $\mathrm{H}(\mathrm{N}) \mathrm{a}$ and $\mathrm{H}(\mathrm{N}) \mathrm{b}$ are the neighboring ammonium protons located at the carboxyl side of the radical.

The extraordinary agreement between theory and experiment confirms that radical model $\mathbf{a 3 a}^{\mathbf{3}}$ corresponds to the 'unstable alanine radical' (species a3a) observed in several EPR experiments. ${ }^{8,9}$

\section{Conversion of a 3 a to a 4 a}

Before the transition from the 'unstable' to the 'stable' alanine radical variant can occur, the detached amino group first needs to become protonated. We already established the presence of this proton in radical species a4a in a previous publication. ${ }^{23}$ In the present work, it turns out that this additional proton effectively drives the transition.

By protonating the amino group of radical model $\mathbf{a 3 a}^{3}$, the net charge of the supercell is neutralized. After energy minimization, this yields radical model $\mathbf{a 4 a}^{\mathbf{1}}, \mathbf{a}$ structure that is virtually identical in conformation to model $\mathbf{a 3} \mathbf{a}^{\mathbf{3}}$, apart from the additional proton (Figure 2). When enough thermal energy is available, $\mathbf{a 4 a}^{\mathbf{1}}$ undergoes an internal rotation to arrive at radical model $\mathbf{a 4 a}^{2}$, which corresponds to the SAR. The internal rotation is in the reverse direction as seen in the transition between $\mathbf{a} 2 \mathbf{a}^{\text {down }}$ and $\mathbf{a 3 a}^{\mathbf{3}}$ : the methyl group of the radical rotates back about the $\mathrm{C} 1$ - 
$\mathrm{C} 2$ axis towards the nearby ammonium. The gain in potential energy by doing so is only about $5 \mathrm{~kJ} / \mathrm{mol}$, but a $45 \mathrm{~kJ} / \mathrm{mol}$ barrier has to be surpassed to reach this minimum. This large barrier, taken together with the slow diffusion of an available proton to the reduced part of the crystal, is the likely explanation for the slow conversion from the 'unstable alanine radical' to the 'stable alanine radical'.

The origin of the proton that attaches to the detached amino group to generate the $S A R$ is not apparent at this moment. A likely source is a radiation-induced oxidized part of the alanine crystal, where a net positive charge is available. However, how exactly a proton is ejected at that site and how it migrates towards the reduced site is entirely unclear, and goes beyond the scope of the present work.

\section{Spectroscopic identification of a $4 a$}

In Table 3, the calculated EPR properties for our $\mathbf{a 4 a}^{2}$ model are compared to the wealth of available experimental data. The results of these calculations differ to some extent from those reported earlier ${ }^{23}$ because a different supercell approach was adopted. The a4a species also has one $\alpha$-type and one $\beta$-type coupling as main spectroscopic features, but the corresponding principal directions are different from those of a3a. Our calculations reproduce the experimental eigenvector directions within $10^{\circ}$ and also yield a good agreement for isotropic and anisotropic values.

In addition, by cooling the $S A R$ to $77 \mathrm{~K}$, Miyagawa and Itoh ${ }^{9}$ were able to stop the methyl group from rotating and measure the hyperfine coupling tensors of the individual methyl protons (indicated $\mathrm{H}(\mathrm{CH} 3) 1-3$ in Table 3 and Figure 2). Their experimental data agrees well with our calculations, except maybe for $\mathrm{H}(\mathrm{CH} 3) 1$ : Miyagawa and Itoh report a tensor with $\alpha$-type character whereas our calculations yield clear $\beta$-character, as would be expected for this proton.

Furthermore, a great number of long-range dipolar couplings have been measured for the a4a species, also taken up in Table 3. Using 2D-HYSCORE spectroscopy, Rakvin and Maltar-Strmecki ${ }^{11}$ have detected the ${ }^{14} \mathrm{~N}$ hyperfine coupling tensor of a nitrogen in the vicinity of the $S A R$, which they suggest to be that of the detached ammonium group. None of the calculated ${ }^{14} \mathrm{~N}$ tensors in our model $\mathbf{a} 4 \mathbf{a}^{2}$ yields a satisfactory agreement with their experiment, although they are likely to be heavily influenced by basis set effects. ${ }^{58}$ The best agreement, mostly for the associated principal directions, is reported in Table 3. However, it puts the detected nitrogen on an alanine molecule neighboring the radical and not on the detached ammonium, as can be seen in Figure SI-2 of the supplementary information. The H(C) dipolar coupling detected by Sagstuen et al. ${ }^{17}$ at room temperature was attributed to a nearby methyl group. Our calculations confirm this analysis: the measurements agree very well with the calculated hyperfine tensor obtained by averaging the individual proton tensors of the methyl group located just below the $S A R$ radical (see Figure SI-2). This is in line with the methyl group being a free rotor at room temperature. EPR measurements at much lower temperatures, then, should be able to make out the different proton contributions as free methyl rotation is inhibited. Indeed, in the $77 \mathrm{~K}$ study by Kuroda and Miyagawa, ${ }^{10}$ two hyperfine tensors are reported that correspond with the protons of this methyl group: $\mathrm{H}(\mathrm{C}) 1$ and $\mathrm{H}(\mathrm{C}) 2$. Two other aliphatic proton dipolar couplings were reported in that work: $\mathrm{H}(\mathrm{C}) 3$ corresponds to a proton of another methyl group below the $S A R$, whereas $\mathrm{H}(\mathrm{C}) 4$ is probably the alpha proton of still another alanine molecule nearby the radical. Two exchangeable couplings, $\mathrm{H}(\mathrm{N}) 1$ and $\mathrm{H}(\mathrm{N}) 2$, can be assigned to protons of the detached ammonium, pointing in the direction of the SAR. 
Overall, the agreement between measured and calculated dipolar couplings adds conclusive support to the validity of our radical model $\mathbf{a} 4 \mathbf{a}^{2}$.

\section{Conclusions}

Using a theoretical approach relying on periodic DFT and NEB calculations, we have simulated the entire reductive radiation-induced transformation pathway of L- $\alpha$ alanine on an atomistic level, yielding unprecedented information on the conformational changes taking place.

The process starts with the electron capture product a0a in which the excess electron is not yet localized. This (unstable) radical species quickly transforms into (unstable) species $\mathbf{a 1 a}^{\text {down }}$, in which the radical center localizes on the carboxyl carbon, concomitant with a "downward" pyramidalization of that group. Proton transfer to the carboxyl group - via the longest hydrogen bond - from the amino group of a neighboring alanine molecule yields radical species $\mathbf{a 2 a}^{\text {down }}$, which is stable up to $150 \mathrm{~K}$. In the temperature interval $150-220 \mathrm{~K}$, (1) the radical deaminates and deprotonates at the carboxyl group, (2) the detached $\mathrm{NH}_{3}$ group undergoes inversion and (3) the methyl group sustains an internal rotation. This results in radical species $\mathbf{a 3 a}^{3}$, which corresponds to what has commonly been called the "unstable alanine radical". Above $220 \mathrm{~K}$, triggered by the attachment of an additional proton on the detached $\mathrm{NH}_{3}$ group, the radical undergoes an internal rotation in the reverse direction. The resulting species $\mathbf{a} 4 \mathbf{a}^{\mathbf{2}}$ - commonly referred to as the "stable alanine radical" - is therefore composed of a negatively charged $\mathrm{CH}_{3} \mathrm{C} \cdot \mathrm{HCO}_{2}{ }^{-}$radical and an associated $\mathrm{NH}_{4}^{+}$ammonium ion, mimicking to some extent the zwitterionic nature of an undamaged alanine molecule in the crystal.

Comparison of calculated EPR spectroscopic properties with experimental data for all stable radicals $\mathbf{a} 2 \mathbf{a}^{\text {down }}, \mathbf{a} 3 \mathbf{a}^{3}$ and $\mathbf{a} \mathbf{4} \mathbf{a}^{2}$ yields a very close agreement, asserting the validity of these models. In addition, the energy profile of the reaction path connecting these states explains why only these species can be observed experimentally. A large energy barrier prevents direct transformation of $\mathbf{a 2 a}^{\text {down }}$ into $\mathbf{a 3 a}^{3}$, requiring sufficient thermal energy. Another energy barrier controls the transformation of $\mathbf{a} 3 \mathbf{a}^{\mathbf{3}}$ into $\mathbf{a} \mathbf{4} \mathbf{a}^{\mathbf{2}}$, but it is most likely the diffusion of an additional proton towards the radical site that needs to be facilitated by thermal energies in excess of $220 \mathrm{~K}$. This proton is proposed to originate from one of the oxidatively damaged radiation-induced sites in the alanine crystal.

\section{Acknowledgements}

This work is supported by the Fund for Scientific Research - Flanders (FWO) and the Research Board of the Ghent University. All computational resources (Stevin Supercomputer Infrastructure) and services were provided by Ghent University, the Hercules Foundation and the Flemish Government - department EWI.

\section{Electronic supplementary information available}

Detailed overview of EPR properties for all model candidates of radical a2a and comparison with experimental data; additional structural representations of radical models $\mathbf{a} 3 \mathbf{a}^{\mathbf{3}}$ and $\mathbf{a} \mathbf{4} \mathbf{a}^{\mathbf{2}}$; an animation with voice over illustrating the reductive radiation chemistry of alanine. 


\section{Tables}

\begin{tabular}{|c|c|c|c|c|c|c|c|c|c|}
\hline & & \multicolumn{5}{|c|}{ Experiment: species a2a } & \multicolumn{3}{|c|}{ Calculation: model $\mathbf{a} 2 \mathbf{a}^{\text {down }}$} \\
\hline & & $\mathrm{A} / \mathrm{g}_{\text {iso }}$ & \multirow{2}{*}{$\frac{\mathrm{A} / \mathrm{g}_{\text {aniso }}}{-77.3}$} & \multicolumn{3}{|c|}{ Principal directions } & \multirow{2}{*}{$\mathrm{A} / \mathrm{g}_{\text {iso }}$} & \multirow{2}{*}{$\frac{\mathrm{A} / \mathrm{g}_{\text {aniso }}}{-63.7}$} & \multirow{2}{*}{$\frac{\text { Angle }}{28}$} \\
\hline ef. & & & & -0.639 & 0.361 & -0.679 & & & \\
\hline \multirow[t]{3}{*}{4} & C1 & 251.3 & -51.2 & -0.674 & 0.163 & -0.721 & \multirow[t]{2}{*}{214.1} & -62.3 & 59 \\
\hline & & & 128.4 & 0.371 & 0.918 & -0.393 & & 126.0 & 13 \\
\hline & & & -9.2 & 0.789 & -0.585 & 0.187 & \multirow{3}{*}{50.1} & -4.8 & 21 \\
\hline \multirow[t]{3}{*}{2} & $\mathrm{H} \alpha$ & 51.0 & 0.0 & 0.581 & 0.612 & -0.537 & & -3.1 & 22 \\
\hline & & & 9.2 & 0.200 & 0.533 & 0.822 & & 7.9 & 7 \\
\hline & & & -13.7 & 0.004 & 0.057 & -0.998 & \multirow{3}{*}{43.4} & -11.0 & 10 \\
\hline \multirow[t]{3}{*}{2} & $\mathrm{H}(\mathrm{O} 1)$ & 43.4 & -1.7 & 0.790 & -0.612 & -0.032 & & -6.2 & 10 \\
\hline & & & 15.4 & 0.613 & 0.788 & 0.048 & & 17.2 & 5 \\
\hline & & & 2.0021 & 0.750 & -0.662 & 0.000 & \multirow{3}{*}{2.0036} & 2.0020 & 26 \\
\hline \multirow[t]{2}{*}{2} & g & 2.0029 & 2.0026 & 0.000 & 0.000 & 1.000 & & 2.0042 & 42 \\
\hline & & & 2.0040 & 0.662 & 0.750 & 0.000 & & 2.0046 & 48 \\
\hline
\end{tabular}

Table 1

Comparison between EPR data of radical species a2a (generated by irradiation and measured below $150 \mathrm{~K}$ ) and calculated properties for radical model a2a ${ }^{\text {down }}$. We chose to use the experimental data of Miyagawa et al. ${ }^{2}$ and Sinclair et al. ${ }^{4}$ as reference. Other, older, data is available, but there are considerable uncertainties in the reported principal directions. Isotropic $\left(\mathrm{A}_{\text {iso }}\right)$ and anisotropic hyperfine couplings $\left(\mathrm{A}_{\text {aniso}}\right)$ are in $\mathrm{MHz}$. Principal directions are given with respect to the orthogonal crystallographic axes $<\mathrm{abc}>$. The last column indicates the angle (in degrees) between corresponding experimental and calculated eigenvector directions. 


\begin{tabular}{|c|c|c|c|c|c|c|c|c|}
\hline & \multicolumn{5}{|c|}{ Experiment: species a3a } & \multicolumn{3}{|c|}{ Calculation: model $\mathbf{a} 3 \mathbf{a}^{3}$} \\
\hline & \multirow{2}{*}{$\mathrm{A}_{\text {iso }}$} & \multirow{2}{*}{$\begin{array}{l}\mathrm{A}_{\text {aniso }} \\
-28.3\end{array}$} & \multicolumn{3}{|c|}{ Principal directions } & \multirow[t]{2}{*}{$\mathrm{A}_{\text {iso }}$} & \multirow{2}{*}{$\frac{A_{\text {aniso }}}{-29.1}$} & \multirow{2}{*}{$\frac{\text { Angle }}{4}$} \\
\hline & & & 0.732 & -0.054 & 0.680 & & & \\
\hline \multirow[t]{3}{*}{$\mathrm{H} \alpha$} & -59.8 & 6.5 & -0.470 & 0.682 & 0.560 & \multirow[t]{2}{*}{-43.1} & -3.1 & 5 \\
\hline & & 21.9 & 0.494 & 0.729 & -0.474 & & 32.2 & 3 \\
\hline & \multirow{3}{*}{66.6} & -3.1 & 0.076 & -0.904 & 0.421 & \multirow{3}{*}{66.0} & -2.8 & 42 \\
\hline \multirow{3}{*}{$\mathrm{H}\left(\mathrm{CH}_{3}\right)$} & & -2.0 & 0.481 & -0.337 & -0.810 & & -2.2 & 42 \\
\hline & & 5.1 & -0.874 & -0.264 & -0.409 & & 4.9 & 7 \\
\hline & & -4.8 & 0.861 & -0.251 & -0.441 & \multirow{3}{*}{1.9} & -3.6 & 49 \\
\hline \multirow[t]{3}{*}{$\mathrm{H}(\mathrm{C}) 1$} & 1.6 & -3.3 & 0.246 & -0.554 & 0.195 & & -2.6 & 72 \\
\hline & & 8.0 & 0.444 & 0.793 & -0.415 & & 6.2 & 2 \\
\hline & & -4.1 & 0.769 & -0.379 & 0.513 & \multirow{3}{*}{0.4} & -2.6 & 3 \\
\hline \multirow{3}{*}{$\mathrm{H}(\mathrm{C}) 2$} & 0.5 & -2.5 & 0.095 & -0.863 & -0.496 & & -2.0 & 14 \\
\hline & & 6.6 & 0.631 & 0.333 & -0.701 & & 4.7 & 2 \\
\hline & & -3.7 & 0.688 & -0.350 & 0.635 & \multirow{3}{*}{-0.1} & -4.1 & 10 \\
\hline \multirow[t]{3}{*}{$\mathrm{H}(\mathrm{N}) \mathrm{a}$} & -0.1 & -1.7 & -0.379 & 0.573 & 0.726 & & -1.4 & 12 \\
\hline & & 5.3 & 0.618 & 0.741 & -0.262 & & 5.5 & 6 \\
\hline & & -2.5 & -0.522 & -0.795 & 0.311 & \multirow{3}{*}{-0.4} & -2.8 & 7 \\
\hline \multirow[t]{2}{*}{$\mathrm{H}(\mathrm{N}) \mathrm{b}$} & -0.4 & -1.4 & 0.825 & -0.563 & -0.055 & & -1.7 & 6 \\
\hline & & 4.0 & 0.218 & 0.228 & 0.949 & & 4.5 & 5 \\
\hline
\end{tabular}

Table 2

Comparison between EPR data of radical species a3a (stable up until $220 \mathrm{~K}$ ) and calculated properties for radical model $\mathbf{a 3 a}^{3}$. All experimental EPR data was taken from Matsuki et al. ${ }^{8}$ Isotropic $\left(\mathrm{A}_{\text {iso }}\right)$ and anisotropic hyperfine couplings $\left(\mathrm{A}_{\text {aniso }}\right)$ are in $\mathrm{MHz}$. Principal directions are given with respect to the orthogonal crystallographic axes $<\mathrm{abc}>$. The last column indicates the angle (in degrees) between corresponding experimental and calculated eigenvector directions. The calculated $\mathrm{H}\left(\mathrm{CH}_{3}\right)$ coupling was obtained by averaging the hyperfine tensors of the individual methyl protons. 


\begin{tabular}{|c|c|c|c|c|c|c|c|c|c|}
\hline \multirow{3}{*}{ Ref. } & & \multicolumn{5}{|c|}{ Experiment: species a4a } & \multicolumn{3}{|c|}{ Calculation: model $\mathbf{a} 4 \mathbf{a}^{2}$} \\
\hline & & $\mathrm{A} / \mathrm{g}_{\text {iso }}$ & $\mathrm{A} / \mathrm{g}_{\text {aniso }}$ & Prin & ipal dire & ions & $\mathrm{A} / \mathrm{g}_{\text {iso }}$ & $\mathrm{A} / \mathrm{g}_{\text {aniso }}$ & Angle \\
\hline & & & -31.8 & 0.467 & 0.416 & 0.780 & \multirow{3}{*}{-42.3} & -30.0 & 2 \\
\hline \multirow[t]{3}{*}{17} & $\mathrm{H} \alpha$ & -56.1 & 3.9 & 0.310 & 0.749 & -0.586 & & -2.5 & 9 \\
\hline & & & 27.9 & 0.828 & -0.515 & -0.221 & & 32.6 & 9 \\
\hline & & & -2.6 & 0.408 & -0.693 & 0.594 & \multirow{3}{*}{71.7} & -2.7 & 6 \\
\hline \multirow[t]{3}{*}{17} & $\mathrm{H}\left(\mathrm{CH}_{3}\right)$ & 69.9 & -2.3 & -0.896 & -0.428 & 0.117 & & -2.0 & 5 \\
\hline & & & 4.8 & -0.173 & 0.581 & 0.795 & & 4.7 & 5 \\
\hline & & & -4.7 & 0.772 & 0.187 & -0.607 & \multirow{3}{*}{2.6} & -3.6 & 63 \\
\hline \multirow[t]{3}{*}{9} & $\mathrm{H}\left(\mathrm{CH}_{3}\right) 1$ & 14.8 & 0.3 & 0.601 & -0.527 & 0.602 & & -3.0 & 63 \\
\hline & & & 4.5 & 0.208 & 0.830 & 0.519 & & 6.7 & 5 \\
\hline & & & -3.9 & -0.140 & 0.686 & -0.714 & \multirow{3}{*}{93.5} & -5.0 & 18 \\
\hline \multirow[t]{3}{*}{9} & $\mathrm{H}\left(\mathrm{CH}_{3}\right) 2$ & 77.6 & -2.5 & 0.799 & 0.522 & 0.348 & & -2.7 & 14 \\
\hline & & & 6.5 & -0.611 & 0.507 & 0.607 & & 7.8 & 12 \\
\hline & & & -4.7 & 1.000 & 0.000 & 0.000 & \multirow{3}{*}{118.8} & -5.3 & 16 \\
\hline \multirow[t]{3}{*}{9} & $\mathrm{H}\left(\mathrm{CH}_{3}\right) 3$ & 121.3 & -3.3 & 0.000 & -1.000 & 0.000 & & -2.8 & 12 \\
\hline & & & 7.9 & 0.000 & 0.000 & 1.000 & & 8.0 & 15 \\
\hline & & & 2.0024 & 0.362 & 0.735 & -0.574 & \multirow{3}{*}{2.0041} & 2.0022 & 5 \\
\hline \multirow[t]{2}{*}{17} & g & 2.0033 & 2.0034 & -0.428 & 0.678 & 0.598 & & 2.0045 & 6 \\
\hline & & & 2.0041 & 0.828 & 0.030 & 0.559 & & 2.0055 & 7 \\
\hline \multirow{3}{*}{11} & & & -1.0 & 0.409 & 0.233 & 0.882 & \multirow{3}{*}{-0.2} & -0.1 & 59 \\
\hline & $\mathrm{N}$ & 1.2 & -0.4 & 0.762 & -0.619 & -0.190 & & -0.1 & 58 \\
\hline & & & 1.4 & -0.502 & -0.750 & 0.431 & & 0.2 & 11 \\
\hline \multirow{3}{*}{17} & & & -2.6 & 0.331 & 0.472 & 0.817 & \multirow{3}{*}{0.6} & -2.5 & 54 \\
\hline & $\mathrm{H}(\mathrm{C})$ & 0.1 & -2.2 & 0.921 & -0.348 & -0.172 & & -1.9 & 54 \\
\hline & & & 4.9 & 0.203 & 0.810 & -0.551 & & 4.3 & 2 \\
\hline \multirow{3}{*}{10} & & & -4.0 & -0.215 & -0.973 & 0.080 & \multirow{3}{*}{0.2} & $\begin{array}{l}-4.3 \\
\end{array}$ & 6 \\
\hline & $\mathrm{H}(\mathrm{N}) 1$ & 0.2 & -1.7 & -0.469 & 0.183 & 0.864 & & -1.3 & 2 \\
\hline & & & 5.7 & 0.856 & -0.114 & 0.495 & & 5.6 & 8 \\
\hline \multirow{3}{*}{10} & & & -2.5 & & & & & -2.7 & \\
\hline & $\mathrm{H}(\mathrm{N}) 2$ & -0.7 & -2.5 & & & & 0.9 & -1.8 & \\
\hline & & & 5.1 & 0.554 & -0.003 & 0.832 & & 4.6 & 3 \\
\hline & & & -5.7 & -0.903 & -0.118 & -0.413 & & -4.4 & 5 \\
\hline 10 & $\mathrm{H}(\mathrm{C}) 1$ & 0.5 & -4.5 & -0.379 & 0.673 & 0.635 & 2.0 & -3.6 & 3 \\
\hline & & & 10.1 & -0.203 & -0.730 & 0.653 & & 8.0 & 5 \\
\hline & & & -2.7 & -0.992 & 0.064 & 0.110 & & -2.3 & 12 \\
\hline 10 & $\mathrm{H}(\mathrm{C}) 2$ & -0.3 & -2.1 & 0.120 & 0.200 & 0.972 & -0.3 & -1.8 & 12 \\
\hline & & & 4.7 & -0.040 & -0.978 & 0.206 & & 4.1 & 2 \\
\hline & & & -1.5 & 0.377 & 0.920 & 0.110 & & -1.6 & 12 \\
\hline 10 & $\mathrm{H}(\mathrm{C}) 3$ & 0.2 & -1.2 & -0.897 & 0.391 & 0.205 & 0.0 & -1.2 & 31 \\
\hline & & & 2.7 & -0.231 & -0.022 & 0.973 & & 2.8 & 8 \\
\hline & & & -2.1 & & & & & -1.9 & \\
\hline 10 & $\mathrm{H}(\mathrm{C}) 4$ & -0.1 & -2.1 & & & & -0.1 & -1.8 & \\
\hline & & & 4.1 & -0.784 & 0.530 & 0.323 & & 3.7 & 2 \\
\hline
\end{tabular}

Table 3

Comparison between EPR data of radical species a4a and calculated properties for radical model $\mathbf{a 4 a}^{2}$. Isotropic $\left(\mathrm{A}_{\mathrm{iso}}\right)$ and anisotropic hyperfine couplings $\left(\mathrm{A}_{\text {aniso }}\right)$ are in $\mathrm{MHz}$. Principal directions are given with respect to the orthogonal crystallographic axes $<\mathrm{abc}>$. The last column indicates the angle (in degrees) between corresponding experimental and calculated eigenvector directions. The calculated $\mathrm{H}\left(\mathrm{CH}_{3}\right)$ and $\mathrm{H}(\mathrm{C})$ couplings were obtained by averaging the corresponding hyperfine tensors of the individual methyl protons. 


\section{Schemes}

Scheme 1

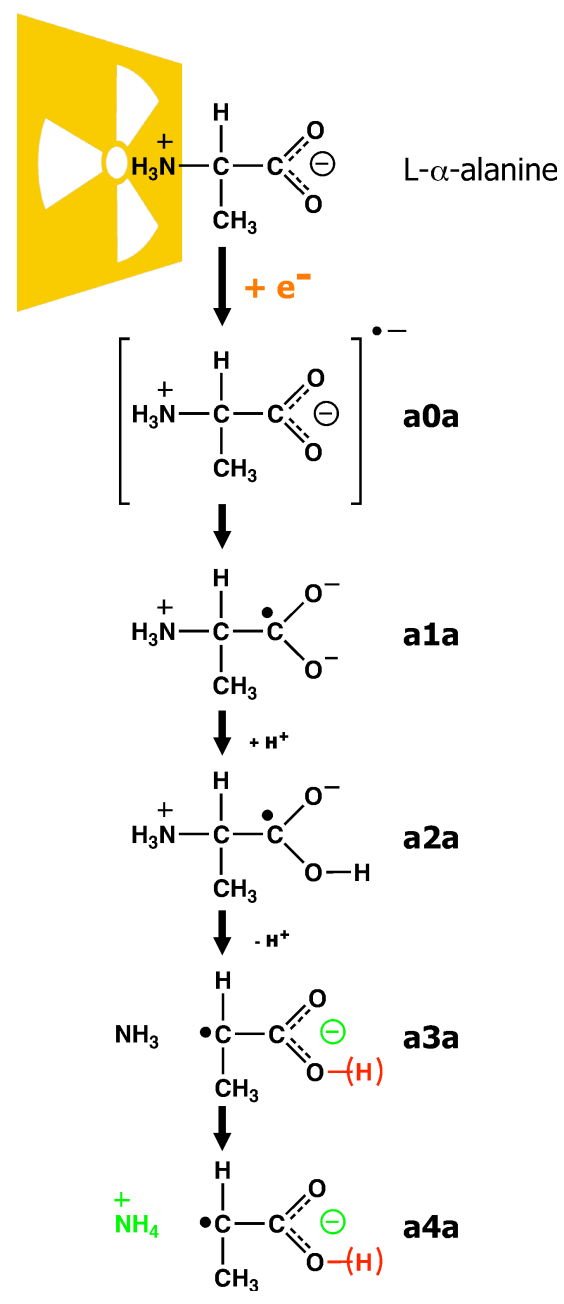

Schematic overview of the reductive radiation-induced formation pathway of the 'Stable Alanine Radical'. Radical species were named in accordance with Sagstuen et al. ${ }^{31} \mathrm{New}$ insights established from the present and previous ${ }^{23}$ works are highlighted in green; prevailing misconceptions with respect to the radical structures are indicated in red: the carboxyl group in a3a and $\mathbf{a 4 a}$ is in fact not protonated. 

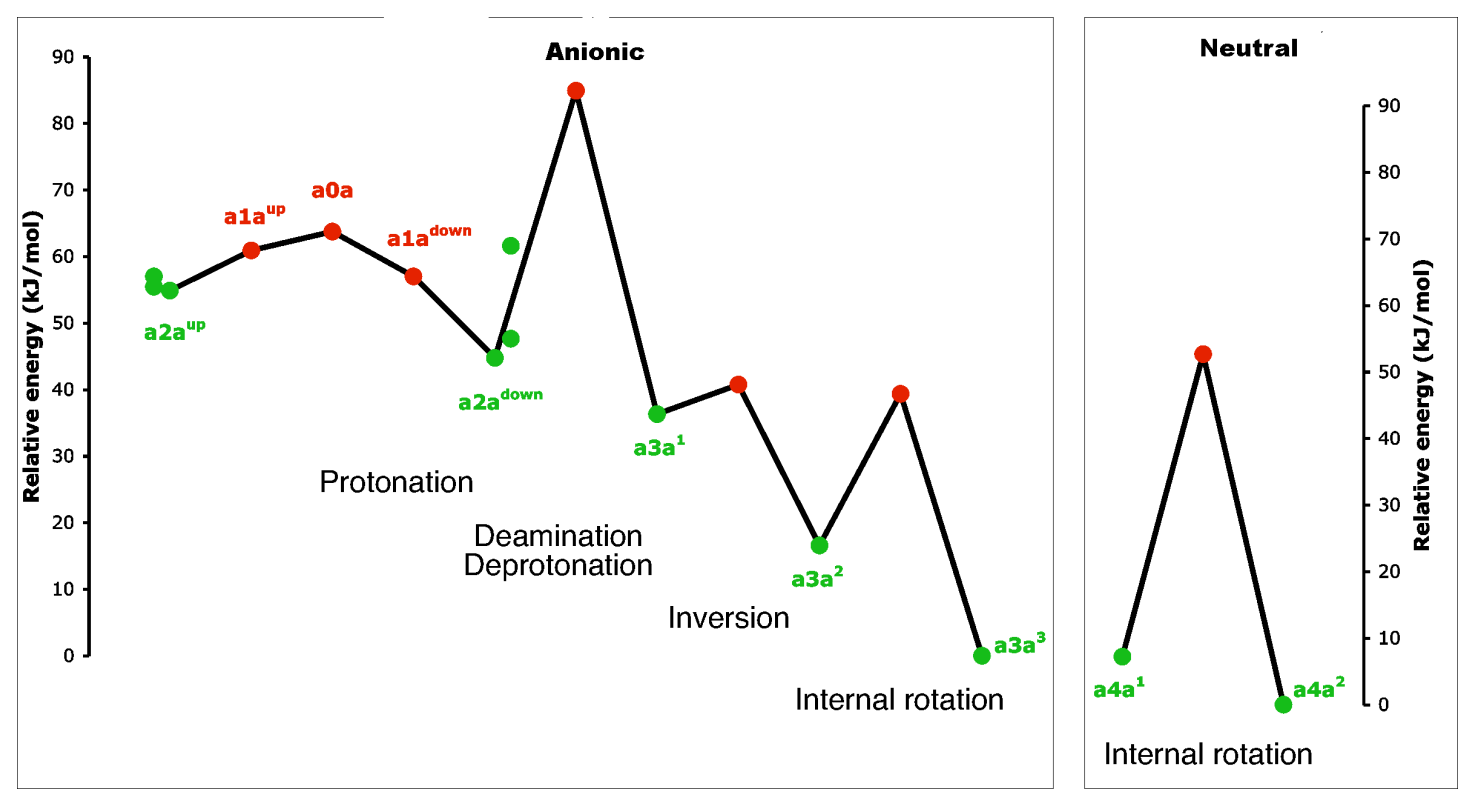

Figure 1

Compound minimal energy path for the radiation-induced formation pathway of the 'Stable Alanine Radical' as obtained from $\mathrm{NEB}^{36,37}$ simulations. Green dots indicate stable minima, red dots indicate structures with maximal potential energy that approximate the transition state. Energies are given in $\mathrm{kJ} / \mathrm{mol}$, relative to the energy of the $\mathbf{a 3 a}^{\mathbf{3}}$ radical structure (-1010.167812 a.u.) for all models obtained in an anionic simulation cell. For the radical structures determined in a neutral, protonated supercell, $\mathbf{a 4 a}^{2}$ was used as a reference (-1010.862387 a.u.). The $\mathbf{a 1 a}^{\text {up }}$ and $\mathbf{a 1 a ^ { \text { down } }}$ species were not obtained from NEB runs but instead by interpolation between a0a and $\mathbf{a} 2 \mathbf{a}^{\text {up }}$, respectively $\mathbf{a} 2 \mathbf{a}^{\text {down }}$. 

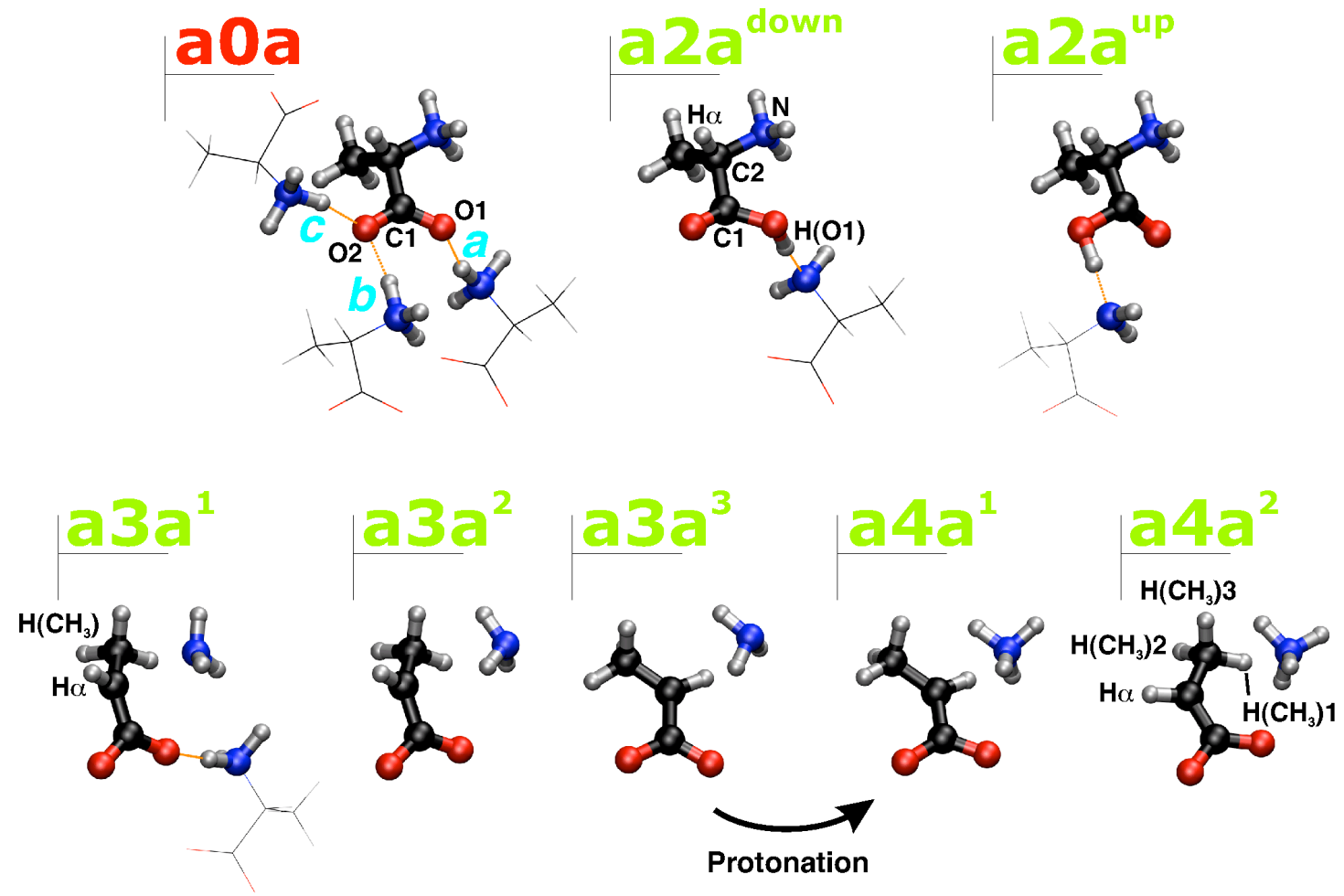

Figure 2

Three-dimensional structure of all minima encountered in the radiation-induced formation pathway of the 'Stable Alanine Radical'. The structure of a0a corresponds to the crystal structure of $\mathrm{L}-\alpha$-alanine ${ }^{34}$ and is given for reference. Several relevant nuclei are explicitly labeled. 3D-renders produced with the aid of VMD. ${ }^{52}$ 


\section{References}

1. E. Sagstuen and E. O. Hole, in Electron Paramagnetic Resonance: A Practitioner's Toolkit, eds. M. Brustolon and E. Giamello, John Wiley \& Sons, 2009, pp. 325-382.

2. I. Miyagawa, N. Tamura, and J. W. Cook, J. Chem. Phys., 1969, 51, 35203528.

3. J. W. Sinclair and M. W. Hanna, J. Phys. Chem., 1967, 71, 84-88.

4. J. W. Sinclair and M. W. Hanna, J. Chem. Phys., 1969, 50, 2125-2129.

5. Minegishi A., Shinozaki Y., and Meshitsuka G., Bull. Chem. Soc. Jpn., 1967, 40, 1549-1550.

6. H. Muto and M. Iwasaki, J. Chem. Phys., 1973, 59, 4821-4829.

7. A. Minegishi, Y. Shinozaki, and G. Meshitsuka, Bull. Chem. Soc. Jpn., 1967, 40, 1271-1272.

8. K. Matsuki and I. Miyagawa, J. Chem. Phys., 1982, 76, 3945-3952.

9. I. Miyagawa and K. Itoh, J. Chem. Phys., 1962, 36, 2157-2163.

10. S. Kuroda and I. Miyagawa, J. Chem. Phys., 1982, 76, 3933-3944.

11. B. Rakvin and N. Maltar-Strmecki, Chem. Phys. Lett., 2005, 415, 161-164.

12. B. Rakvin, N. Maltar-Strmecki, C. M. Ramsey, and N. S. Dalal, J. Chem. Phys., 2004, 120, 6665-6673.

13. B. Rakvin, N. Maltar-Strmecki, D. Kattnig, and G. Grampp, Mol. Phys., 2007, 105, 2087-2094.

14. N. Maltar-Strmecki and B. Rakvin, Appl. Radiat. Isot., 2005, 63, 375-380.

15. N. Maltar-Strmecki and B. Rakvin, Spectrochim. Acta Part a-Molecular Biomol. Spectrosc., 2006, 63, 784-787.

16. J. R. Morton and A. Horsfield, J. Chem. Phys., 1961, 35, 1142-1143.

17. E. Sagstuen, E. O. Hole, S. R. Haugedal, and W. H. Nelson, J. Phys. Chem. A, 1997, 101, 9763-9772.

18. B. Rakvin, N. Maltar-Strmecki, and K. Nakagawa, Radiat. Meas., 2007, 42, 1469-1474.

19. E. Malinen, M. Z. Heydari, E. Sagstuen, and E. O. Hole, Radiat. Res., 2003, 159, 23-32. 
20. E. Malinen, E. A. Hult, E. O. Hole, and E. Sagstuen, Radiat. Res., 2003, 159, 149-153.

21. M. Z. Heydari, E. Malinen, E. O. Hole, and E. Sagstuen, J. Phys. Chem. A, 2002, 106, 8971-8977.

22. G. Vanhaelewyn, S. A. Amira, W. Mondelaers, and F. J. Callens, Spectrochim. Acta Part a-Molecular Biomol. Spectrosc., 2000, 56, 387-397.

23. E. Pauwels, H. De Cooman, M. Waroquier, E. O. Hole, and E. Sagstuen, Phys. Chem. Chem. Phys., 2010, 12, 8733-8736.

24. E. Pauwels, V. Van Speybroeck, P. Lahorte, and M. Waroquier, J. Phys. Chem. $A, 2001,105,8794-8804$.

25. A. Gil, S. Simon, L. Rodriguez-Santiago, J. Bertran, and M. Sodupe, J. Chem. Theory Comput., 2007, 3, 2210-2220.

26. T. L. Petrenko, J. Phys. Chem. A, 2002, 106, 149-156.

27. P. Lahorte, F. De Proft, G. Vanhaelewyn, B. Masschaele, P. Cauwels, F. Callens, P. Geerlings, and W. Mondelaers, J. Phys. Chem. A, 1999, 103, 66506657.

28. F. Q. Ban, S. D. Wetmore, and R. J. Boyd, J. Phys. Chem. A, 1999, 103, 43034308.

29. C. Adamo, V. Barone, and A. Fortunelli, J. Chem. Phys., 1995, 102, 384-393.

30. A. van Roggen, L. van Roggen, and W. Gordy, Bull. Am. Pys. Soc., 1956, 1, 266.

31. E. Sagstuen, A. Sanderud, and E. O. Hole, Radiat. Res., 2004, 162, 112-119.

32. K. Itoh and I. Miyagawa, J. Mol. Struct., 1988, 190, 85-91.

33. http://www.cp2k.org.

34. M. S. Lehmann, T. F. Koetzle, and W. C. Hamilton, J. Am. Chem. Soc., 1972, 94, 2657-2660.

35. R. H. Byrd, P. H. Lu, J. Nocedal, and C. Y. Zhu, Siam J. Sci. Comput., 1995, 16, 1190-1208.

36. G. Henkelman, B. P. Uberuaga, and H. Jonsson, J. Chem. Phys., 2000, 113, 9901-9904.

37. G. Henkelman and H. Jonsson, J. Chem. Phys., 2000, 113, 9978-9985.

38. P. Pulay, Chem. Phys. Lett., 1980, 73, 393-398. 
39. S. G. Aalbergsjo, E. Pauwels, H. De Cooman, E. O. Hole, and E. Sagstuen, Phys. Chem. Chem. Phys., 2013, 15, 9615-9619.

40. A. D. Becke, Phys. Rev. A, 1988, 38, 3098-3100.

41. C. T. Lee, W. T. Yang, and R. G. Parr, Phys. Rev. B, 1988, 37, 785-789.

42. G. Lippert, J. Hutter, and M. Parrinello, Mol. Phys., 1997, 92, 477-487.

43. J. VandeVondele and J. Hutter, J. Chem. Phys., 2007, 127, 9.

44. S. Goedecker, M. Teter, and J. Hutter, Phys. Rev. B, 1996, 54, 1703-1710.

45. C. Hartwigsen, S. Goedecker, and J. Hutter, Phys. Rev. B, 1998, 58, 36413662.

46. R. Declerck, E. Pauwels, V. Van Speybroeck, and M. Waroquier, Phys. Rev. B, 2006, 74, 8 .

47. V. Weber, M. Iannuzzi, S. Giani, J. Hutter, R. Declerck, and M. Waroquier, $J$. Chem. Phys., 2009, 131, 11.

48. M. Krack and M. Parrinello, Phys. Chem. Chem. Phys., 2000, 2, 2105-2112.

49. N. Godbout, D. R. Salahub, J. Andzelm, and E. Wimmer, Can. J. Chem. Can. Chim., 1992, 70, 560-571.

50. E. Pauwels, R. Declerck, V. Van Speybroeck, and M. Waroquier, Radiat. Res., 2008, 169, 8-18.

51. M. A. Tarpan, H. De Cooman, E. Sagstuen, M. Waroquier, and F. Callens, Phys. Chem. Chem. Phys., 2011, 13, 11294-11302.

52. W. Humphrey, A. Dalke, and K. Schulten, J. Mol. Graph., 1996, 14, 33-38, $27-28$.

53. K. T. Ohman and E. Sagstuen, J. Phys. Chem. A, 2008, 112, 4284-4293.

54. K. T. Ohman, A. Sanderud, E. O. Hole, and E. Sagstuen, J. Phys. Chem. A, 2006, 110, 9585-9596.

55. E. Sagstuen, E. O. Hole, and A. Lund, Radiat. Phys. Chem., 2012, 81, 168 179.

56. E. Pauwels, V. Van Speybroeck, and M. Waroquier, J. Phys. Chem. A, 2004, 108, 11321-11332.

57. E. Pauwels, T. Verstraelen, H. De Cooman, V. Van Speybroeck, and M. Waroquier, J. Phys. Chem. B, 2008, 112, 7618-7630. 
58. L. Hermosilla, P. Calle, J. M. G. de la Vega, and C. Sieiro, J. Phys. Chem. A, 2006, 110, 13600-13608. 